\title{
On the origin of giant pulses in radio pulsars
}

\begin{abstract}
S. A. Petrova
Institute of Radio Astronomy, 4, Chervonopraporna St., Kharkov, 61002 Ukraine

e-mail: rai@ira.kharkov.ua

Received 30 January 2004 / Accepted 11 May 2004

Abstract. Induced Compton scattering of radio emission off the particles of the ultrarelativistic highly magnetized plasma of pulsar magnetosphere is considered. The scattering between the photon beams of substantially differing frequencies and orientations appears to result in photon transfer to the higher frequency. With the decreasing spectrum of pulsar radio emission, this may imply a significant amplification of the higher-frequency intensity. A detailed analysis shows that the rays of widely spaced frequencies can have different orientations, at least at certain locations in the pulsar magnetosphere. The numerical estimates confirm the possibility of an efficient beam-to-beam scattering in the pulsars known as giant pulse sources. Thus, the intensity amplification on account of induced scattering of the lower-frequency radiation is suggested to underlie the giant pulse phenomenon. A huge pulse-to-pulse scatter in the observed intensities of the giant pulse sources (over more than three orders of magnitude) testifies to significant variations of the efficiency of amplification. If this variability is attributed to the fluctuations of both the particle number density and the incident intensity and the latter are considered as the partially correlated Gaussian random quantities, one arrives at the power-law distribution of strong pulses in intensity. The formation of the giant pulse substructure, the broad-band spectral behaviour of the scattering efficiency and the link of giant pulses to the high-energy emission are discussed as well.
\end{abstract}

Key words. plasmas - waves - scattering - pulsars: general - pulsars: individual: the Crab pulsar, PSR B1937+21

\section{Introduction}

\subsection{Observational evidence}

The single-pulse intensities of radio pulsars typically fluctuate within a few times the average. However, several pulsars are known to emit occasional giant pulses with intensities up to a few thousand times the average. The giant pulses usually constitute a tiny fraction of the total of pulses and do not affect the average profile. The most distinctive feature of giant pulses is their power-law distribution in intensity, in contrast to the Gaussian or exponential distribution characteristic of the normal pulses (e.g. Lundgren et al. 1995; Kinkhabwala $\&$ Thorsett 2000). Giant pulses have specific profiles, with fast rise and exponential decay (e.g. Sallmen et al. 1999), and are narrower than a normal pulse, the strongest ones tending to be the narrowest (e.g. Cognard et al. 1996). For the Crab pulsar it is found that giant pulses often consist of several components with widths ranging down to a few nanoseconds (Hankins 1996, 2000; Hankins et al. 2003). These nanopulses are also characterized by fast rise and exponential decay and the strongest of them tend to be the shortest. Recent observations of PSR B1937+21 (Popov \& Stappers 2003) also give indirect evidence in favour of a temporal structure of giant pulses at nanosecond timescales.

Giant pulses occur over a broad range of radio frequencies, but the correlation bandwidth is not large. As is found by Popov \& Stappers (2003), giant pulses of PSR B1937+21 are not simultaneous in the interval $\Delta v / v \sim 0.5$. In the Crab pulsar, the nanostructure is correlated only over $\Delta v / v \sim 0.2$ (Eilek et al. 2002), although giant pulses are seen simultaneously at widely spaced frequencies.

Giant radio pulses are commonly thought to be related to high-energy emission. Although a drastic increase of intensity is peculiar only to the radio frequency range, there are some indications of that link. Firstly, the locations of giant pulses of PSR B1937+21 in the pulse window accord with the high-energy profiles better than with the average radio profile (Cusumano et al. 2003). Secondly, the optical pulses of the Crab pulsar, which accompany giant pulses, are 3\% brighter than those which coincide with the normal pulses (Shearer et al. 2003).

At present the nature of giant pulses remains obscure. The most important problem concerns the source of energy responsible for the occasional huge amplification of radiation and the statistics of energy release underlying the characteristic powerlaw distribution of giant pulses in intensity. The nanostructure, which is thought to be a fundamental feature of giant pulse emission, presents another puzzle.

\subsection{Interpretation of nanostructure}

On a qualitative level, apart from the scaling factors, the nanostructure of giant pulses looks similar to the microstructure in normal pulsars (e.g. Hankins 1996), hinting at a similar 
origin of the two phenomena. The observed micro/nano-pulses can be interpreted in two different ways. Firstly, they can be attributed to the spatial variations in relativistically streaming pulsar plasma, which are likely to affect the emission process and further propagation of radio waves. Then, in the case of microstructure, the characteristic scale of the plasma inhomogeneities is $\sim 3 \mathrm{~km}$ (Popov et al. 2002), whereas the giant nanopulses result from the plasma clumps $\sim 3 \mathrm{~m}$ in size (Hankins et al. 2003). These plasma inhomogeneities are usually associated with the soliton-like structures which indeed may arise in the strongly turbulent plasma of pulsar magnetosphere (e.g. Asseo et al. 1990; Weatherall 1998).

In the alternative interpretation, the micro/nano-pulses are believed to result from the inhomogeneity of the angular pattern of the emission cone, which manifests as the pulsar rotates. This interpretation is strongly supported by observations: the timescales of microstructure are proportional to the pulsar period (Hankins 1996; Popov et al. 2002), testifying to the same characteristic angular scale of the emission pattern. According to the most straightforward considerations, the angular width of the micro/nano-pulses is related to the opening angle of the relativistically beamed radiation. However, this requires too large Lorentz-factors of the secondary plasma, $\gamma \sim 10^{4}-10^{7}$, and seems inadmissible.

As is shown in Petrova (2004), propagation in pulsar magnetosphere can lead to an additional beaming of radiation. Induced Compton scattering off the secondary particles is found to cause substantial focusing of the radio photons, so that the resultant beam appears squeezed up to a few hundred times. It has been demonstrated that the focusing effect can account for the timescales of the observed microsturcture as well as for its polarization properties. In the present paper, we extend the study of induced Compton scattering in application to pulsar magnetospheres and suggest an explanation for giant pulses and their substructure.

\subsection{Statement of the problem}

We consider induced scattering of relativistically beamed radiation off the particles of the ultrarelativistic highly magnetized plasma streaming along the open magnetic lines in the pulsar magnetosphere. In the rest frame of the scattering particles, the photon frequency is practically unaltered in the course of the scattering. Correspondingly, in the laboratory frame, the scattering inside a narrow beam, $\theta \lesssim 1 / \gamma$, can change the photon frequency only slightly and acts mainly to redistribute the photon directions within the beam, whereas the beam intensity remains approximately constant. However, below it will be shown that significant amplification of the beam intensity can also be the case in pulsar magnetosphere. Although at a given frequency pulsar radiation is undoubtedly concentrated into a narrow beam, the beams of significantly differing frequencies can have substantially different orientations with respect to the velocity of the scattering particles. This may arise from the difference in the locations of the emission regions or from differential action of propagation effects in pulsar plasma. Then the induced scattering between the photon states with widely spaced frequencies may become possible. Exact analysis of the kinetic equation shows that the photons are mainly transferred to the higher-frequency beam, the total intensity of the two beams being constant. With the decreasing spectrum of pulsar radiation, this may imply significant intensity amplification of the higher-frequency beam. If, say, the frequencies of the two beams are in the ratio of $\sim 1: 10$ and the spectral index is $\approx 3$, the intensity of the higher-frequency beam can be enhanced up to $\sim 1000$ times. Thus, the induced scattering of photons between widely spaced frequencies, if efficient, can indeed account for the energetics of giant pulses.

Numerical estimates show that in the pulsars known as giant pulse sources the optical depth to the induced scattering, $\Gamma$, may run into values large enough to provide substantial amplification of intensity. The low frequency of occurence of giant pulses hints at a significant variability of $\Gamma$. According to the simplest considerations, the scattering efficiency varies because of pulse-to-pulse fluctuations of both the number density of the scattering particles and the intensity of incident radiation. If they are regarded as partially correlated Gaussian random quantities, one arrives at the power-law distribution of the pulses markedly amplified in intensity $(\Gamma>1)$, which is the salient feature of the observed giant pulses.

Apart from the beam-to-beam scattering, the induced scattering within each beam acts to focus the photons inside the beam. The focusing effect coincident with giant pulses is likely to be stronger because of larger plasma densities and incident intensities as well as because of the possible extra-beaming in the course of beam-to-beam scattering. Correspondingly, giant pulse emission is expected to be focused into separate components with timescales shorter than those of the microstructure in other pulsars.

Thus, the basic features of giant pulses and their substructure can be explained in terms of induced Compton scattering of pulsar radiation off the particles of the plasma flow. The plan of the paper is as follows. In Sect. 2 we solve the system of kinetic equations describing the photon transfer between two narrow beams as a result of induced Compton scattering in ultrarelativistic highly magnetized plasma. The solution obtained is applied to the case of pulsars: we specify the location of the region of beam-to-beam scattering and estimate the scattering efficiency. In Sect. 3 we develop a simplified statistical model of intensity amplification which provides the power-law distribution of giant pulses in intensity. In Sect. 4 the results of our study are discussed in the context of the observations. Section 5 contains a brief summary.

\section{Induced scattering of radio emission in pulsar plasma}

\subsection{General equations}

Let a beam of radio photons propagate through the ultrarelativistic highly magnetized electron-positron plasma of a pulsar magnetosphere. The plasma particles stream along the open magnetic lines with Lorentz factors $\sim 100$. The brightness temperatures of pulsar radio emission are known to be extremely high, $T_{\mathrm{b}} \sim 10^{25}-10^{30} \mathrm{~K}$, and therefore the induced scattering 
of radio photons off the plasma particles is expected to play a marked role. Numerical estimates show that the optical depth to this process can indeed be significant at pulsar conditions (e.g. Blandford \& Scharlemann 1976; Lyubarskii \& Petrova 1996).

The presence of a strong magnetic field can substantially affect the scattering process, changing both the particle recoil and the scattering cross-section. This happens on condition that the photon frequency in the rest frame of the scattering particles is much less than the electron gyro-frequency, $\omega^{\prime} \ll \frac{e B}{m c}$. The induced scattering in the limit of a strong magnetic field takes place in the inner magnetosphere, where the number density of the scattering particles is the largest, and hence dominates the scattering in the weak field (Lyubarskii \& Petrova 1996). The kinetic equation describing the evolution of the photon occupation numbers, $n(\boldsymbol{k})$, as a result of induced Compton scattering off the ultrarelativistic particles in an infinitely strong magnetic field is written as (e.g. Eq. (30) in Blandford \& Scharlemann 1976):

$$
\begin{aligned}
\frac{\partial n(k, \theta, \phi)}{\partial t} & +c \frac{\partial n(k, \theta, \phi)}{\partial r}=\frac{\hbar}{m} n_{\mathrm{e}} r_{\mathrm{e}}^{2} n(k, \theta, \phi) \\
& \times \int \frac{\sin ^{2} \theta \sin ^{2} \theta_{1}}{\gamma^{6} \eta^{3} \eta_{1}^{3} \beta^{2}}\left\{\frac{1}{\gamma^{3}}\left(\frac{\eta_{1}-\eta}{\eta_{1}}\right)^{2} \frac{\partial}{\partial k}\left[k_{1}^{2} n_{1}\left(k_{1}, \theta_{1}, \phi_{1}\right)\right]\right. \\
& \left.+\frac{6 k \eta^{2}\left(\eta_{1}-\eta\right) n_{1}\left(k_{1}, \theta_{1}, \phi_{1}\right)}{\gamma \eta_{1}^{2}}\left[1-\frac{1}{2 \gamma^{2}}\left(\frac{\eta_{1}+\eta}{\eta \eta_{1}}\right)\right]\right\} \\
& \times \theta_{1} \mathrm{~d} \theta_{1} \mathrm{~d} \phi_{1} .
\end{aligned}
$$

Here $(k, \theta, \phi)$ are the spherical coordinates of the wavevector $\boldsymbol{k}$ in the system whose polar axis coincides with the axis of the photon beam and the plane containing the magnetic field vector corresponds to the zero azimuth; $r_{\mathrm{e}}$ is the classical electron radius, $n_{\mathrm{e}}$ the particle number density, $\beta$ the particle velocity in units of $c, \eta \equiv 1-\beta \cos \vartheta_{\mathrm{b}}$, with $\vartheta_{\mathrm{b}}$ being the angle between the wavevector and the particle velocity, and the integral is taken over the solid angle of the photons participating in the scattering. Note that Eq. (1) corresponds to the case of cold plasma. Although the secondary particles in the pulsar magnetosphere are known to have a large spread in Lorentz factors, the main contribution to the right-hand side of the kinetic equation comes from the particles with Lorentz factors close to some characteristic value. Since an exact form of the particle distribution function is still unknown, Eq. (1) presents a reasonable approximation. The results for the realistic case of hot plasma are believed to be qualitatively the same.

In the above kinetic equation

$k \eta=k_{1} \eta_{1}$

signifying that in the rest frame of the scattering particles the photon frequency is unchanged. Then, in the case of a narrow photon beam with the opening angle $\lesssim 1 / \gamma, \eta \approx \eta_{1}$ and in the laboratory frame the photons are scattered only between the states with close frequencies, so that the scattering acts mainly to redistribute the photon orientations inside the beam. As is found in Petrova (2004), given a large enough angle between the beam axis and the ambient magnetic field, $\psi \gg 1 / \gamma$, the photons can be strongly focused toward the direction closest to the magnetic field vector, whereas the beam energy remains almost unaltered.

Significant energy transfer can be expected from the induced scattering between substantially differing photon states which still obey the relationship (2). Although at a given frequency pulsar radio photons are concentrated into a narrow beam, beams of widely spaced frequencies can have different orientations. As is shown in Sect. 2.2, this situation can occur in pulsars. So, let us consider two narrow photon beams, which propagate quasi-transversely with respect to the magnetic field, $\psi_{a, b} \gg 1 / \gamma$, so that $\eta_{a, b} \approx \psi_{a, b}^{2} / 2$, and $\psi_{b}-\psi_{a} \gg 1 / \gamma$. Then the variation of the photon intensities, $i_{v_{a, b}}\left(\theta_{a, b}, \phi_{a, b}\right)=$ $2 h v_{a, b}^{3} n_{a, b}\left(\boldsymbol{k}_{a, b}\right) / c^{2}$, in the course of induced scattering is given by the following set of equations:

$$
\begin{aligned}
\frac{\partial i_{v_{a}}\left(\theta_{a}, \phi_{a}\right)}{\partial a r}= & i_{v_{a}}\left(\theta_{a}, \phi_{a}\right) \int i_{v_{b}}\left(\theta_{b}, \phi_{b}\right)\left(\eta_{b}-\eta_{a}\right) \mathrm{d} \Omega_{b} \\
& +\frac{\eta_{b}^{3}}{\eta_{a}^{3}} i_{v_{a}}\left(\theta_{a}, \phi_{a}\right) \int i_{v_{a}}\left(\theta_{a_{1}}, \phi_{a_{1}}\right)\left(\eta_{a_{1}}-\eta_{a}\right) \mathrm{d} \Omega_{a_{1}} \\
\frac{\partial i_{v_{b}}\left(\theta_{b}, \phi_{b}\right)}{\partial a r}= & i_{v_{b}}\left(\theta_{b}, \phi_{b}\right) \int i_{v_{a}}\left(\theta_{a}, \phi_{a}\right)\left(\eta_{a}-\eta_{b}\right) \mathrm{d} \Omega_{a} \\
& +\frac{\eta_{a}^{3}}{\eta_{b}^{3}} i_{v_{b}}\left(\theta_{b}, \phi_{b}\right) \int i_{v_{b}}\left(\theta_{b_{1}}, \phi_{b_{1}}\right)\left(\eta_{b_{1}}-\eta_{b}\right) \mathrm{d} \Omega_{b_{1}}
\end{aligned}
$$

$a \equiv \frac{192 n_{\mathrm{e}} r_{\mathrm{e}}^{2}}{m v_{a}^{2} \gamma^{7}} \psi_{a}^{-6} \psi_{b}^{-2}$.

Note that in Eqs. (3) the terms corresponding to the first addend on the right-hand side of Eq. (1) are not included because they are $(\psi \gamma)^{2}$ less than the others. We concentrate on the stationary case and omit the derivatives $\partial i_{a, b} / \partial t$. The first terms in Eqs. (3) describe the photon scattering between the two beams, whereas the second ones correspond to the scattering inside the beams. To study the energy transfer between the beams let us introduce the total intensity of a beam,

$I_{a, b}=\int i_{v_{a, b}}\left(\theta_{a, b}, \phi_{a, b}\right) \theta_{a, b} \mathrm{~d} \theta_{a, b} \mathrm{~d} \phi_{a, b}$,

and integrate Eqs. (3) over the solid angles. Then we obtain:

$$
\frac{\partial I_{a}}{\partial a r}=I_{a} I_{b}\left(\eta_{b}-\eta_{a}\right)
$$

$\frac{\partial I_{b}}{\partial a r}=-I_{a} I_{b}\left(\eta_{b}-\eta_{a}\right)$.

Keeping in mind Eq. (2), one can see that the photons are transferred from the low-frequency beam to the high-frequency one. In addition, it is evident that the system of Eqs. (6) has the first integral,

$I_{a}+I_{b}=I=$ constant.

Hence, it can be reduced to the form

$\frac{\partial}{\partial a r}\left(\frac{I_{a}}{I_{b}}\right)=I \frac{I_{a}}{I_{b}}\left(\eta_{b}-\eta_{a}\right)$ 
and the solution is written as

$\frac{I_{a}}{I_{b}}=\frac{I_{a_{0}}}{I_{b_{0}}} \exp \left(I\left(\eta_{b}-\eta_{a}\right) a r\right)$.

Making use of Eq. (7), one can find finally:

$I_{a}=\frac{I \frac{I_{a_{0}}}{I_{b_{0}}} \exp \left(I\left(\eta_{b}-\eta_{a}\right) a r\right)}{1+\frac{I_{a_{0}}}{I_{b_{0}}} \exp \left(I\left(\eta_{b}-\eta_{a}\right) a r\right)}$,

$I_{b}=\frac{I}{1+\frac{I_{a_{0}}}{I_{b_{0}}} \exp \left(I\left(\eta_{b}-\eta_{a}\right) a r\right)}$.

Thus, the energy transfer between the beams is determined by the parameter $\Gamma \equiv I\left(\eta_{b}-\eta_{a}\right) a r$. It is easy to see that at $\Gamma \rightarrow \infty$ (and $\eta_{b}>\eta_{a}$ ) $I_{a} \rightarrow I$. In the present paper, we are particularly interested in the scattering between widely spaced frequencies (i.e. $v_{a} \gg v_{b}$ and, consequently, given the decreasing spectrum of radiation, $\left.I_{a_{0}} \ll I_{b_{0}}\right)$ at moderately large $\Gamma$, so that $\frac{I_{a_{0}}}{I_{b_{0}}} \mathrm{e}^{\Gamma} \ll 1$, in which case

$I_{a} \approx I_{a_{0}} \mathrm{e}^{\Gamma} \quad$ and $\quad I_{b} \approx I$.

In this approximation, the high-frequency intensity can increase significantly, but still remains much less than $I$.

\subsection{Location of the scattering region}

Now let us turn to the question of whether the induced scattering between widely spaced frequencies is indeed possible in the pulsar magnetosphere, i.e. whether the condition

$v_{a} \psi_{a}^{2} / 2=v_{b} \psi_{b}^{2} / 2$

can be satisfied for $v_{a} \gg v_{b}$. Generally speaking, at a fixed point in the open field line tube the rays of distinct frequencies can be inclined at different angles to the ambient magnetic field either as a result of radius-to-frequency mapping, which makes them propagate along different paths in the rotating magnetosphere, or because of refraction, which is known to be frequency-dependent.

To begin with, we consider the simplest situation, when the rays are emitted along the field lines of the dipolar magnetic field and propagate straight in the non-rotating magnetosphere. If $\left(r_{0}, \chi_{0}\right)$ are the polar coordinates of the emission point in the system with the polar axis along the magnetic axis, the polar angle of the ray at an altitude $r$ is given by

$\chi=\chi_{0}\left(\frac{3}{2}-\frac{r_{0}}{2 r}\right)$

where it is taken into account that the wavevector makes the angle $\theta_{0}=3 \chi_{0} / 2$ with the magnetic axis. Then the angle between the wavevector and the ambient magnetic field, $\psi=$ $3 x / 2-3 \chi_{0} / 2$, is

$\psi=\frac{3}{2} \chi \frac{r-r_{0}}{3 r-r_{0}}$

Hence, $\psi$ is a function of $r_{0}$ and, given the radius-to-frequency mapping, depends on the frequency. However, it is easy to see that $\frac{\mathrm{d} \psi}{\mathrm{d} r_{0}}=\frac{-3 \chi r}{\left(3 r-r_{0}\right)^{2}}<0$, i.e. the larger emission altitudes imply simultaneously the lower frequencies and the smaller $\psi$, so that the condition (12) is never fulfilled for $v_{a} \neq v_{b}$.

For a more realistic situation, it is reasonable to include pulsar rotation. One of the giant pulse sources, PSR B1937+21, has the shortest known period, $P=1.56 \mathrm{~ms}$, in which case the light cylinder radius, $r_{L}=5 \times 10^{9} \mathrm{P} \mathrm{cm}$, is only about 10 times the radius of a neutron star, $r_{\star} \approx 10^{6} \mathrm{~cm}$. One can expect that the contribution of the rotational effect, $\sim \frac{r-r_{0}}{r_{L}}$, is particularly significant in this pulsar. Let us choose the spherical coordinate system with the polar axis along the magnetic axis and the zero azimuth defined by the meridional plane containing the rotational axis. If a ray is emitted at the point $\left(r_{0}, \chi_{0}, \phi_{0}\right)$ tangentially to the magnetic field line and propagates along a straight line in a rapidly rotating magnetosphere, at large enough distances, $\frac{r-r_{0}}{r_{L}} \gg \chi_{0}$, the ray trajectory is given by

$\chi=\frac{r-r_{0}}{r_{L}} \sin \zeta-\chi_{0} \sin \phi_{0}\left(\frac{3}{2}-\frac{r_{0}}{2 r}\right), \quad \sin \phi \approx-1$,

where $\zeta$ is the angle between the rotational and magnetic axes. In this approximation, the inclination of the wavevector to the magnetic axis, $\theta$, is written as

$\theta=\frac{r-r_{0}}{r_{L}} \sin \zeta-\theta_{0} \sin \phi_{0}$,

with $\theta_{0}=3 \chi_{0} / 2$. Then at $r \gg r_{0}$ the angle $\psi$ between the wavevector and the ambient magnetic field, $\psi=3 \chi / 2-\theta$, is reduced to the form:

$\psi=\frac{\chi}{2}+\frac{r_{0}}{3 r_{L}} \sin \zeta$.

Hence, $\psi$ depends on the emission altitude, $r_{0}$, and, consequently, on the ray frequency. Apparently, this dependence is prominent only at very small $\chi, \chi \ll\left(r_{0} / r_{L}\right) \sin \zeta$, i.e. at the locations close enough to the magnetic axis. According to Eq. (17), $\psi$ is a decreasing function of frequency, so that the necessary condition of induced scattering (Eq. (12)) can be satisfied for widely spaced frequencies. For example, given the theoretically predicted law, $r_{0} \propto v^{-2 / 3}$, for the frequency ratio $v_{b} / v_{a}=0.1 \mathrm{Eq}$. (12) is fulfilled at $\chi=0.5\left(r_{0_{a}} / r_{L}\right) \sin \zeta=$ $0.1\left(r_{0_{b}} / r_{L}\right) \sin \zeta$. Then one can find that $\chi_{0} \sim \theta_{0_{a}} \sim\left(r / r_{L}\right) \sin \zeta$ and $\theta_{a} \approx 0$.

Thus, in the laboratory frame, the radiation which can be subject to amplification as a result of induced scattering is roughly co-directed with the magnetic axis. Recall that because of relativistic aberration the profile maximum leaves behind the projection of the magnetic axis defined by $\theta_{a}=0$. In case of PSR B1937+21, in both the main pulse and interpulse, giant pulses indeed markedly lag behind the pulse maxima, and the time delay is always practically constant: $\tau_{\mathrm{MP}}=58.2 \mu \mathrm{s}$ and $\tau_{\mathrm{IP}}=66.6 \mu$ s (e.g. Popov \& Stappers 2003). Then we obtain the following estimate: $r / r_{L} \sim \theta_{0_{a}} \approx 2 \pi \tau / P \sim 0.2$, which seems to be quite realistic. It should be noted that in reality the concrete form of the radius-to-frequency mapping is likely to differ substantially from the assumed law and our quantitative results can be somewhat changed, however, the main qualitative conclusions that the scattering region lies close enough to the magnetic axis and the loci of giant pulses in the pulse 
window roughly correspond to the projection of the magnetic axis are believed to be unaltered. Thus, the specific longitudinal location of giant pulses of PSR B1937+21 can be attributed to the rotational effect, which provides the appropriate geometry of the beams of distinct frequencies, so that the induced Compton scattering can result in pronounced amplification of the higher-frequency radiation.

In view of the significant role of the rotational effect, it should be mentioned that in the previous subsection we have not differentiated between the frame corotating with the magnetosphere and the laboratory frame. However, the directions of the wavevector and the particle velocity are transformed between these frames in the same manner, so that the angle $\psi$ between them remains unaltered to the first order in $r / r_{L}$. In this approximation, all the other quantities entering Eqs. (1)-(11) are also unchanged and hence our results are still applicable for both of these frames.

For the Crab pulsar, another well-known source of giant pulses, its period is about 20 times longer than that of PSR B1937+21 and, correspondingly, the rotational effect is much weaker over most of its magnetosphere and can hardly account for the proper geometry of the photon beams participating in the induced scattering. The observationally established fact that giant pulses of the Crab pulsar have no preferential longitudinal location and can be found throughout the main pulse and interpulse (for more details see Cordes et al. 2003) also argues against the dominant role of the rotational effect. At the same time, the difference in the orientations of the rays of widely spaced frequencies is still expected for typical pulsars and confirmed indirectly by observations. Firstly, the structure of pulse profiles can drastically evolve with frequency (Rankin 1983). Secondly, the spatial distribution of intensity, which can be derived for the closest pulsars by means of interstellar interferometry, appears qualitatively different at various frequencies (cf. Wolszczan et al. 1988; Gupta et al. 1999).

Both these features can be explained in terms of the frequency-dependent refraction of radio waves in pulsar plasma (Petrova 2000, 2001a). Although a detailed picture of refraction depends strongly on the assumed distribution of the plasma, one can conclude that the necessary condition for induced scattering between widely spaced frequencies (Eq. (12)) can be satisfied. The high-frequency rays can be strongly declined toward the edges of the open field line tube; further along the trajectory, as refraction ceases, they propagate straight and finally their extra-inclination to the ambient magnetic field is compensated for by divergence of the field lines. The numerical calculations for typical pulsar parameters confirm the possibility of quasi-longitudinal propagation of the high-frequency rays with respect to the magnetic field (see Fig. 1 in Petrova $2001 b$ ). On the other hand, the low-frequency rays are not affected by refraction and their inclination to the ambient magnetic field increases monotonically along the trajectory, so that the case of $\psi_{a} \ll \psi_{b}$ at $v_{a} \gg v_{b}$ seems to be relevant to the magnetosphere of a typical pulsar. If so, the scattering region should lie at distances of the order of the emission altitude, whereas its structure across the tube, which is determined by the instanteneous distribution of the plasma, can be strongly variable.

\subsection{Efficiency of induced scattering}

\subsubsection{Beam-to-beam scattering}

Above we have found that the induced scattering between the beams of substantially differing frequencies can indeed take place in pulsar magnetosphere. Now we are going to examine whether it can be strong enough to account for a significant amplification of radiation. Because of the small frequency of occurence of giant pulses, the beam-to-beam scattering is expected to be efficient only occasionally. In the present paper, it is assumed that in the pulsar case the optical depth to induced scattering varies from pulse to pulse owing to the fluctuations of the plasma number density, $n_{\mathrm{e}}$, and original intensity, $I_{a_{0}}\left(=I_{b_{0}}\left(v_{a} / v_{b}\right)^{-\alpha}\right)$. As is shown in Sect. 3, the observed statistics of giant pulses can be reproduced at $\Gamma \sim 0.1 \frac{n_{\mathrm{e}}}{\left\langle n_{\mathrm{e}}\right\rangle} \frac{I_{a_{0}}}{\left\langle I_{a_{0}}\right\rangle}$, where $\left\langle n_{\mathrm{e}}\right\rangle$ and $\left\langle I_{a_{0}}\right\rangle$ are the average values of $n_{\mathrm{e}}$ and $I_{a_{0}}$. Then the giant pulses (i.e. those which correspond to $\Gamma \gtrsim 1$ ) occur at $n_{\mathrm{e}} \gtrsim 3\left\langle n_{\mathrm{e}}\right\rangle$ and $I_{a_{0}} \gtrsim 3\left\langle I_{a_{0}}\right\rangle$, whereas for the normal pulses (at $n_{\mathrm{e}} \approx\left\langle n_{\mathrm{e}}\right\rangle$ and $\left.I_{a_{0}} \approx\left\langle I_{a_{0}}\right\rangle\right) \Gamma \sim 0.1$. Thus, one should estimate whether the optical depth to the induced scattering can indeed be $\sim 0.1$ for the typical parameters of the pulsars known as giant pulse sources.

Taking $I \approx I_{b}$ (cf. Eq. (11)) and $\eta_{b}-\eta_{a} \approx \psi_{b}^{2} / 2$, we have:

$\Gamma=\frac{96 n_{\mathrm{e}} r_{\mathrm{e}}^{2}}{m v_{a}^{2} \gamma}\left(\psi_{a} \gamma\right)^{-6} I_{b} \Delta r$,

where $\Delta r$ is the length of the scattering region. The intensity of radiation can be presented as

$I_{b}=I_{b_{0}}\left(\frac{v_{b}}{v_{0}}\right)^{-\alpha}$.

Here $v_{0} \approx 100 \mathrm{MHz}$ and

$I_{b_{0}}=\frac{L}{v_{0} \pi r^{2}(w / 2)^{2}}$

where $L$ is the total radio luminosity of a pulsar and $w$ the pulse width in the angular measure. It is convenient to introduce the multiplicity factor of the plasma, $\kappa=N P c e / B$, which is the plasma number density normalized to the Goldreich-Julian density. Then one can find:

$$
\begin{aligned}
\Gamma= & 1.7 P \frac{L}{10^{30} \mathrm{erg} \mathrm{s}^{-1}} \frac{B_{\star}}{10^{12} \mathrm{G}} \frac{\kappa}{10^{2}}\left(\frac{\tau}{10^{-3} \mathrm{~s}}\right)^{-2}\left(\frac{\gamma}{10^{2}}\right)^{-1} \\
& \times\left(\frac{v_{b}}{10^{8} \mathrm{~Hz}}\right)^{-\alpha}\left(\frac{v_{a}}{10^{9} \mathrm{~Hz}}\right)^{-2}\left(\frac{\psi_{a}}{0.3} \frac{\gamma}{10^{2}}\right)^{-6} \\
& \times\left(\frac{r}{10^{8} \mathrm{~cm}}\right)^{-5}\left(\frac{\Delta r}{10^{8} \mathrm{~cm}}\right)
\end{aligned}
$$

where $B_{\star}$ is the magnetic field strength at the surface of the neutron star, the stellar radius is taken to be $10^{6} \mathrm{~cm}$ and $\tau$ is the pulse width in the temporal measure, $\tau=P w / 2 \pi$. All the quantities are normalized to their typical values. In particular, the multiplicity factor and the characteristic Lorentz factor of the plasma roughly correspond to those given by the modern theories of the pulsar pair creation cascade (e.g. Hibschman \& Arons 2001a,b). 
According to Taylor et al. (1993), for the Crab pulsar and PSR B1937+21 the 400-MHz luminosities equal $1.4 \times 10^{29}$ and $1.1 \times 10^{29} \mathrm{erg} \mathrm{s}^{-1}$, respectively. Taking into account that $L \propto$ $v^{1-\alpha}$ (with $\alpha=2.8$ and 2.6) we obtain that at $v_{0}=100 \mathrm{MHz}$ $L=1.7 \times 10^{30}$ and $10^{30} \mathrm{erg} \mathrm{s}^{-1}$, respectively. Then for the Crab pulsar $\left(P=0.033 \mathrm{~s}, \log B_{\star}=12.6\right.$ and $\left.\tau=2 \mathrm{~ms}\right) \Gamma=0.1$ on condition that all the rest quantites entering Eq. (21) have the standard values equal to the normalizing factors. In case of PSR B1937 $+21\left(P=1.56 \mathrm{~ms}, \log B_{\star}=8.6\right.$ and $\left.\tau=0.1 \mathrm{~ms}\right)$ $\Gamma=0.1$ at $r \sim 10^{7} \mathrm{~cm}$ and $\Delta r \sim r$. Thus, for the ostensible giant pulse sources the estimate $\Gamma \sim 0.1$ is quite realistic.

The two pulsars considered can be regarded as prototypes of the two distinct classes of giant pulse sources, representing the young pulsars and the shortest-period millisecond pulsars. The characteristics of PSR B0540-69 and its giant pulses (Johnston \& Romani 2003) are very close to those of the Crab pulsar, whereas another known giant pulse source, PSR B1821-24 (Romani \& Johnston 2001), is similar to PSR B1937+21. Note that the above estimates and the suggested mechanism of giant pulses on the whole are also applicable to PSR B0540-69 and PSR B1821-24. Another two pulsars recognized as giant pulse sources, PSR B1112+50 (Ershov \& Kuzmin 2003) and PSR B0031-07 (Kuzmin et al. 2004), appear to be quite different from the others: they have long periods and can show series of neighbouring giant pulses - a phenomenon never seen before. Interpretation of this peculiar case is beyond the framework of the present paper.

If one applies Eq. (21) to predicting another giant pulse sources, it is evident that, first of all, such sources should be characterized by large luminosities. Note that the Crab pulsar and PSR B1937+21 have the largest known radio luminosities (see, e.g. Malov \& Malov 1997). Because of the possibility of a significant period dependence of almost all of the quantities entering Eq. (21), it is difficult to conclude about the actual period dependence of $\Gamma$ and find out whether the giant pulse sources should really have the largest magnetic field strengths at the light cylinder, i.e. whether $\Gamma \propto B_{\star} P^{-3}$. For example, if the altitude of the scattering region constitutes a certain fraction of the light cylinder, $r \propto r_{L}$, and $\Delta r \propto r$, whereas the period dependence of the rest quantities is negligible (or compensated for), Eq. (21) yields $\Gamma \propto B_{\star} P^{-3}$. Unfortunately, the numerical value of $\Gamma$ depends crucially on the location of the scattering region and the scattering geometry and therefore does not allow firm conclusions as to the efficiency of the beam-to-beam scattering in a given pulsar.

The possibility of giant pulses depends not only on the scattering depth (i.e. on the efficiency of photon transfer) but also on the intensity ratio, $I_{b_{0}} / I_{a_{0}}$, which determines the maximum level of amplification at $\Gamma \rightarrow \infty$. Apparently, for a reasonable frequency ratio, $v_{a} / v_{b}$, the observed amplification (up to a few thousand times) requires a steep enough (average) spectrum of pulsar radio emission. The giant pulse sources known at present indeed have steep spectra. Moreover, the observations testify to the correlation between the spectral index and the level of amplification in giant pulses: PSR B0540-69 has an extremely steep spectrum, $\alpha<-4.4$ and shows an amplification $>5000$, which exceeds that ever observed in the Crab pulsar (Johnston \& Romani 2003).

\subsubsection{Scattering inside the beam}

Let us turn to the induced scattering inside a narrow beam, in which case the photons are transferred between the close frequencies and tend to be focused in a certain direction within the beam. This process accompanies the beam-to-beam scattering and, being independent of the condition (12), takes place throughout the beam trajectory in pulsar plasma. It is most efficient deep inside the magnetosphere, where the number density of the scattering particles is the largest. As is found in Petrova (2004), at the conditions relevant to pulsar magnetosphere, induced scattering inside the beam can lead to a strong focusing of photons. It is the process that is suggested to underlie the microstructure observed in a number of pulsars. Despite the lack of observational data on the microstructure in the normal pulses of giant pulse sources, we estimate the scattering efficiency in the Crab pulsar in order to conclude whether this process can account for the substructure of its giant pulses.

The optical depth to induced scattering inside the beam is given by Eq. (14) in Petrova (2004):

$$
\begin{aligned}
\Gamma_{i}= & 340 P \frac{L}{10^{28} \mathrm{erg} \mathrm{s}^{-1}} \frac{B_{\star}}{10^{12} \mathrm{G}} \frac{\kappa}{10^{2}}\left(\frac{\tau}{10^{-3} \mathrm{~s}}\right)^{-2}\left(\frac{\gamma}{10^{2}}\right)^{-1} \\
& \times\left(\frac{v_{a}}{10^{8} \mathrm{~Hz}}\right)^{-\alpha-2}\left(\frac{\psi_{a}}{10^{-1}} \frac{\gamma}{10^{2}}\right)^{-7} \\
& \times\left(\frac{r}{10^{8} \mathrm{~cm}}\right)^{-4}
\end{aligned}
$$

Using the parameters of the Crab pulsar listed above and taking $v_{a}=10^{9} \mathrm{~Hz}, r=3 \times 10^{7} \mathrm{~cm}$ and $\psi=0.05$, we obtain $\Gamma_{i}=474$. This value refers to normal pulses and is roughly consistent with the characteristic optical depth $\left(\Gamma_{i} \sim 200\right)$ required by the observed minimum timescales of microstructure in other pulsars (for more details see Petrova 2004).

Similar to the optical depth to the beam-to-beam scattering, $\Gamma_{i}$ should fluctuate because of pulse-to-pulse variations of the plasma density and the incident intensity. As is discussed in Sect. 2.3.1, for the normal pulses $\Gamma \sim 0.1$, whereas the maximum observed amplification, $\sim 2000$, corresponds to $\Gamma_{\max } \approx 7$. Hence, one can take $\Gamma_{i_{\max }} \approx 70 \Gamma_{i}$. Taking the beam angular width $w_{0} \sim 1 / \gamma$ and keeping in mind that because of focusing $w \sim w_{0} / \Gamma_{i}$, one can estimate the timescale of the substructure in the strongest giant pulses as $\tau \sim P /\left(2 \pi \gamma \Gamma_{i_{\max }}\right) \sim 10^{-9} \mathrm{~s}$. Thus, induced scattering inside the beam can indeed account for the structural details of giant pulses down to the nanosecond timescales. Note that the scattering between the beams of widely spaced frequencies can result in further focusing inside the beams.

\section{Statistical model for intensity amplification in giant pulses}

To study statistics of intensity amplification as a result of induced scattering let us turn to a simplified model, where the scattering efficiency is changed because of pulse-to-pulse fluctuations of the plasma number density, $n_{\mathrm{e}}$, and the original intensity, $I_{a_{0}}$. These quantities are assumed to be random and obey the bivariate Gaussian distribution. For the normalized 
quantities, $y=I_{a_{0}} /\left\langle I_{a_{0}}\right\rangle$ and $z=n_{\mathrm{e}} /\left\langle n_{\mathrm{e}}\right\rangle$, the density of the probability distribution is written as

$$
\begin{aligned}
p(y, z)= & \frac{1}{2 \pi \sigma_{y} \sigma_{z} \sqrt{1-\mu^{2}}} \exp \left\{-\frac{1}{2\left(1-\mu^{2}\right)}\right. \\
& \left.\times\left[\frac{(y-1)^{2}}{\sigma_{y}^{2}}-2 \mu \frac{(y-1)(z-1)}{\sigma_{y} \sigma_{z}}+\frac{(z-1)^{2}}{\sigma_{z}^{2}}\right]\right\},
\end{aligned}
$$

where $\mu$ is the correlation coefficient (note that the original intensity of radiation and the plasma density can indeed be markedly correlated, since the radio emission process is associated with pulsar plasma). In accordance with Eqs. (11) and (18), the final intensity, $i=I_{a} / I_{a_{0}}$, is given by $i=$ $y \exp (\varepsilon y z)$. To satisfy the observed frequency of occurence of giant pulses it is taken that $\varepsilon \ll 1$. Then for the normal pulses $(y, z \lesssim 1) \varepsilon y z \ll 1$ and $i \approx y$, i.e. the distribution of final intensity approximately follows the Gaussian distribution of the original intensity. Giant pulses correspond to the opposite limit, $\varepsilon y z \gg 1$, in which case $i \approx \exp (\varepsilon y z)$. To obtain the density of the probability distribution of giant pulse intensities let us first find the probability density function of the product $x=y z$ at $y, z \gg 1$.

According to the definition, the distribution function of the product $x=y z$ (at $y, z \gg 1)$ has the form

$F(x)=\int_{0}^{\infty} \int_{0}^{x / z} p(y, z) \mathrm{d} y \mathrm{~d} z$,

and the distribution density, $p(x)=\partial F / \partial x$, is reduced to

$p(x)=\int_{0}^{\infty} \frac{1}{z} p(x / z, z) \mathrm{d} z$.

Incorporating Eq. (22) into Eq. (24) and making use of the relation (Gradshteyn \& Ryzhik 1980)

$\int_{0}^{\infty} x^{\nu-1} \exp \left(-\beta x^{p}-\gamma x^{-p}\right) \mathrm{d} x=\frac{2}{p}\left(\frac{\gamma}{\beta}\right)^{v / 2 p} K_{v / p}(2 \sqrt{\beta \gamma})$,

one can obtain:

$$
\begin{aligned}
p(x)= & \frac{1}{2 \pi \sigma_{y} \sigma_{z} \sqrt{1-\mu^{2}}} \exp \left(\frac{\mu x}{\sigma_{y} \sigma_{z}\left(1-\mu^{2}\right)}\right) \\
& \times K_{0}\left(\frac{x}{\sigma_{y} \sigma_{z}\left(1-\mu^{2}\right)}\right),
\end{aligned}
$$

where $K_{0}(\cdot)$ is the modified Bessel function of the zeroth order. Taking into account its asymptotic behaviour,

$K_{0}(z) \approx \sqrt{\frac{\pi}{2 z}} \mathrm{e}^{-z}$ at $z \rightarrow \infty$,

we come to

$p(x)=\frac{1}{2 \sqrt{2 \pi \sigma_{y} \sigma_{z} x}} \exp \left(-\frac{x}{\sigma_{y} \sigma_{z}(1+\mu)}\right)$.

The density of the probability distribution of $i$ is given by $f(i)=p(x(i)) \mathrm{d} x / \mathrm{d} i$; hence, incorporating $x=\ln i / \varepsilon$ and $\mathrm{d} x / \mathrm{d} i=(\varepsilon i)^{-1}$, we find finally:

$f(i)=\frac{1}{2 \sqrt{2 \pi \varepsilon \sigma_{y} \sigma_{z} \ln i}} i^{-\frac{1}{\varepsilon \sigma_{y} \sigma_{z}(1+\mu)}-1}$.

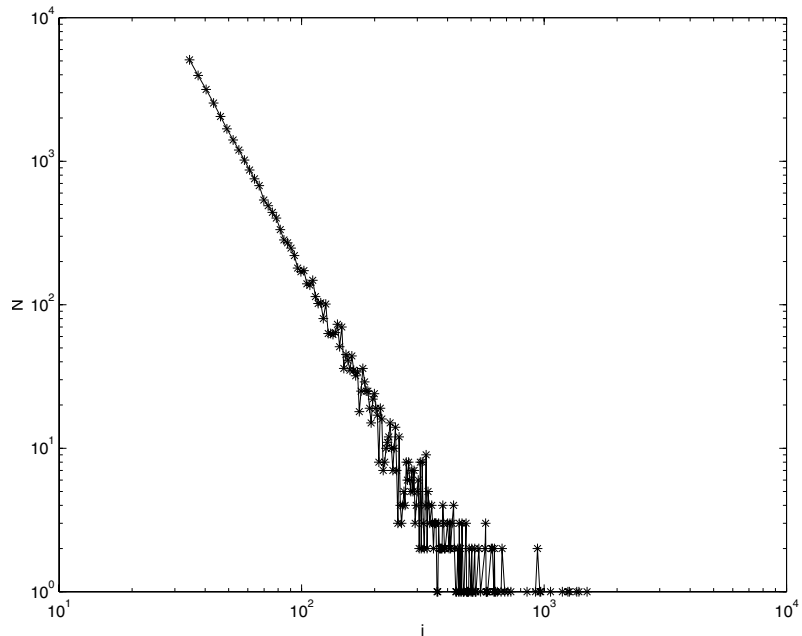

Fig. 1. The simulated distribution of giant pulses in intensity $(i \geq 33)$; $N_{\text {total }}=10^{7}, \varepsilon=0.1, \sigma_{y}=\sigma_{z}=1.3, \mu=0.9$.

One can see that at $i \gg 1 f(i)$ has approximately a power-law form with the exponent determined by the characteristic scattering depth, $\varepsilon$, the variances, $\sigma_{y, z}$, and the correlation coefficient, $\mu$. Thus, our simplified model reproduces the power-law intensity distribution for the strongly amplified pulses, which is known to be a salient feature of the statistical behaviour of giant pulses (Lundgren et al. 1995; Kinkhabwala \& Thorsett 2000).

Given that the giant pulses are defined as those happening at $\varepsilon x \geq \xi$ (where $\xi$ is a certain number larger than unity), their frequency of occurrence can be written as

$$
\begin{aligned}
\frac{N_{\mathrm{GP}}}{N_{\text {total }}} & \equiv \int_{\xi / \varepsilon}^{\infty} p(x) \mathrm{d} x \\
& =\frac{1}{\sqrt{2 \pi}} \sqrt{\frac{1+\mu}{2}} \int_{\sqrt{\frac{2 \xi}{\varepsilon \sigma_{y} \sigma_{z}(1+\mu)}}}^{\infty} \exp \left(-v^{2} / 2\right) \mathrm{d} v .
\end{aligned}
$$

It is evident that $\frac{N_{\mathrm{GP}}}{N_{\mathrm{total}}}$ is chiefly determined by the same parameter $u=\varepsilon \sigma_{y} \sigma_{z}(1+\mu)$, which enters the exponent in Eq. (27), and only slightly depends on $\mu$. Hence, the observed distribution of giant pulse intensities does not allow us to differentiate between the distinct sets of co-factors resulting in the same value of $u$.

As an example of our model, we have simulated the pulse distribution in final intensities by means of computer modelling. Using a generator of random numbers, we have constructed $10^{7}$ pairs $(y, z)$ which obey a bivariate Gaussian distribution (Eq. (22)). The histogram of the distribution of giant pulses in final intensities, $i=y \exp (\varepsilon y z)(i \geq 33)$, is presented in Fig. 1. Here we have assumed $\varepsilon=0.1, \sigma_{y}=\sigma_{z}=1.3$ and $\mu=0.9$. The histogram obtained is well fitted by the power law with the exponent -3.26 ; the total number of giant pulses in the sample considered is $N_{\mathrm{GP}}=30542$. These parameters and the histogram on the whole are in a good agreement with those presented in Lundgren et al. (1995) based on the observational data for the Crab pulsar. 


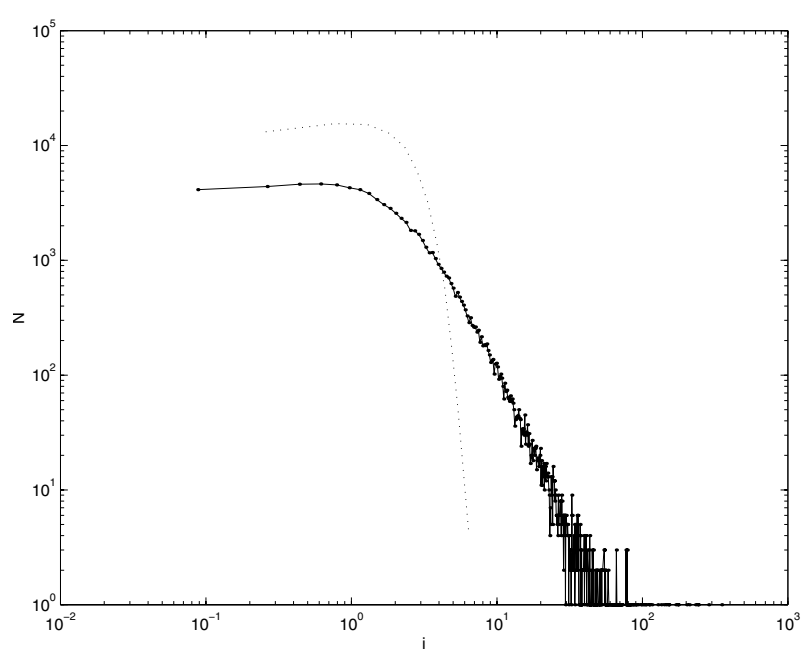

Fig. 2. The simulated distribution of the total of pulses in intensity; $N_{\text {total }}=10^{5}, \varepsilon=0.1, \sigma_{y}=\sigma_{z}=1.3, \mu=0.9$. The dotted line shows the assumed Gaussian distribution in original intensity.

Figure 2 shows the histogram of the complete distribution in pulse intensities, $i=y \exp (\varepsilon y z)$, for all conceivable $y$ and $z$; here we have used a set of $10^{5}$ pairs $(y, z)$ and the same parameters $\varepsilon, \sigma_{y, z}$ and $\mu$. The distribution of pulses in original intensity, $y$, is plotted by the dotted line. In Fig. 2 the powerlaw segment corresponding to giant pulses is also present, but its slope is substantially less, -2.5 . The exponent of the power law describing the distribution of giant pulses in intensity depends markedly on the total number of the pulses considered, and only at $N_{\text {total }} \rightarrow \infty$ the slope approaches the theoretically predicted value, $-\left[\varepsilon \sigma_{y} \sigma_{z}(1+\mu)\right]^{-1}-1$ (i.e. -4.11 for the parameters assumed above). In general, the form of the intensity distribution demonstrated in Fig. 2 needs observational confirmation, since as a rule in the observed distributions a significant part is dominated by noise.

\section{Discussion}

Within the framework of our interpretation, giant pulses arise as a result of induced Compton scattering of pulsar radiation off the plasma particles. We have considered the scattering between the photon beams of widely spaced frequencies and distinct orientations, in which case the photon transfer from the lower-frequency beam to the higher-frequency one is possible. It has been found that such a situation can indeed be the case in the pulsar magnetosphere and the scattering can occasionally become strong enough to provide a significant energy transfer between the beams. The resultant intensities of the amplified radiation appear to match those observed in giant pulses. It has been demonstrated that the observed statistics of intensity amplification in giant pulses can be explained in terms of a simple model, where the pulse-to-pulse variations of the optical depth to induced scattering are attributed to the fluctuations of the particle number density and the original intensity and the latter are considered as partially correlated Gaussian random quantities. In this case, for the pulses subject to substantial amplification the distribution in intensity has a characteristic power-law form, which is indeed peculiar to the observed distribution of giant pulses.

Because of the non-linear increase of intensity, $i=$ $y \exp (\varepsilon y z)$, even a huge amplification $\left(i \sim 10^{3}-10^{4}\right)$ results from moderate (within an order of magnitude) variations of the plasma density and original intensity. This is supported by the lack of significant changes in the observed high-energy radiation concurrent with giant radio pulses. The increase of the gamma-ray flux in the $50-200 \mathrm{keV}$ range is limited to a factor of 2.5, testifying against substantial changes in both the efficiency of the pair creation process and the final characteristics of the outflowing plasma (Lundgren et al. 1995). The optical emission, which is usually associated with the incoherent synchrotron emission of the plasma particles, is also only $3 \%$ brighter than average (Shearer et al. 2003). Thus, giant pulses are not associated with drastic electrodynamic changes in the pulsar magnetosphere. Moreover, as the intensity amplification is attributed to the non-linear propagation effect, both the normal and giant pulses are expected to be generated by the same emission mechanism, which is confirmed by the lack of a marked time delay between them (Lundgren et al. 1995).

The induced scattering between the beams of widely spaced frequencies is thought to be confined to certain regions of the open field line tube, with the scattering geometry varying substantially across each of them. Then, keeping in mind the exponential form of intensity growth, $i=i_{0} \mathrm{e}^{\Gamma}$, one can estimate the width of giant pulse as $w_{\mathrm{GP}} \sim w / \Gamma$. Hence, for the strongest pulses $(\Gamma \gg 1)$ the larger intensities imply shorter durations, which is in agreement with the observed trend (e.g. Cognard et al. 1996). Recall, however, that the observed giant pulse shapes are commonly supposed to be smeared by the scattering in the ISM or in the pulsar surroundings (Sallmen et al. 1999).

Apart from the beam-to-beam scattering, the photons are certainly subject to induced scattering inside the beam. This process takes place all over the ray path in the plasma and is most efficient close to the emission region, where the number density of the scattering particles is the largest. As is found in Petrova (2004), the scattering inside the beam can be significant in a variety of pulsars and lead to photon focusing, which is believed to underlie pulsar microsturcture. For the giant pulses the focusing effect is believed to be even stronger. Firstly, the scattering inside the beam is enhanced because of somewhat larger plasma densities and original intensities peculiar to giant pulses. Secondly, the beam-to-beam scattering can lead to further focusing of radiation. As is found in Sect. 2.3.2, in giant pulses the focusing can account for the structural details at the timescales down to a nanosecond.

Such a substructure of giant pulses is indeed present in the Crab pulsar (Eilek et al. 2002; Hankins et al. 2003). Note that taking into account the focusing effect can remove the problem of exceedingly high brightness temperatures of the nanopulses. Besides that, the origin of the giant subpulses as a result of focusing has observational support. As is found by Eilek et al. (2002), the plot of the subpulse peak intensity versus width shows an upper envelope, which is consistent with the constant energy of the subpulse, $I_{0} \tau=$ constant. Then one can suppose that the variability of the intensities below the envelope is due 
to changes in the beam-to-beam scattering efficiency, which result in various levels of true amplification of radiation, whereas the envelope itself corresponds to the subpulses subject to the maximum amplification along with the focusing of different efficiency.

The giant pulses of PSR B1937+21 do not show a substructure, even though there is indirect evidence of the presence of subpulses with the widths $\sim 10-100 \mathrm{~ns}$ (Popov \& Stappers 2003). This can be easily understood if the substructure of a giant pulse is related to the angular pattern of photon beams (set up by the focusing effect): the period 20 times smaller than that of the Crab pulsar implies accordingly shorter temporal scales of the subpulses.

Proceeding from the explicit frequency dependence of the scattering efficiency (see Eq. (21)), one can estimate the bandwidth of the amplified radiation as $\Delta v / v \sim 1 / \Gamma$. Taking into account that the maximum observed amplification is about a few thousand times and, correspondingly, $\Gamma_{\max } \approx 7$, we have $\Delta v / v \gtrsim 0.1$, which accords well with the correlation bandwidth of the substructure in the Crab pulsar (Eilek et al. 2002). At the same time, the conditions for efficient beam-to-beam scattering can be satisfied simultaneously in a number of distinct regions in the open field line tube and different frequencies can be amplified independently. As a result, a complicated picture of the broad-band activity of giant pulses may arise, being determined by the global frequency dependence of the scattering efficiency, which is contained implicitly in the geometrical parameters of the scattering region, $\psi$ and $r$.

Note that some information on the broad-band frequency dependence of $\Gamma$ can be derived from the observed dual-frequency single-pulse spectra of giant pulses. Given that the intensities at the two frequencies are written as $i_{1,2}=i_{0_{1,2}} \exp \left(\varepsilon_{1,2} n_{\mathrm{e}} i_{0_{1,2}}\right)$, where $\varepsilon_{1} / \varepsilon_{2}=\left(v_{1} / v_{2}\right)^{l}$ and $i_{0_{1}} / i_{0_{2}}=\left(v_{1} / v_{2}\right)^{-\alpha_{0}}$, the observed spectral index, $\alpha=$ $-\ln \left(i_{1} / i_{2}\right) / \ln \left(v_{1} / v_{2}\right)$, is reduced to

$-\alpha=-\alpha_{0}+\frac{\varepsilon_{1} i_{0_{1}} n_{\mathrm{e}}}{\ln \left(v_{1} / v_{2}\right)}\left[1-\left(v_{1} / v_{2}\right)^{\alpha_{0}-l}\right]$.

One can see that the sign of the shift of $\alpha$ from the original value, $\alpha_{0}$, depends on the sign of $l-\alpha_{0}$, whereas the shift itself varies from pulse to pulse because of the random fluctuations of $n_{\mathrm{e}}$ and $i_{0_{1}}$. Proceeding from Eq. (26), which gives the distribution of the probability density for the product of the Gaussian random quantities, one can find that the histogram of the final spectral indices, $\alpha$, should show an exponential tail on one side of the maximum (in addition to the symmetrical smearing of the histogram on account of noise).

The histograms of such a shape are indeed peculiar to the observed single-pulse spectra, testifying to the frequencydependent amplification. In the case of the Crab pulsar, the distribution of $\alpha$ has a tail towards the steeper indices (Hankins 2000), so that intensity amplification is more efficient at the lower frequencies and, correspondingly, $l-\alpha_{0}<0$. In contrast, in PSR B1133+16 the histogram of the single-pulse spectral indices exhibits a tail toward the flatter $\alpha$ (Kramer et al. 2003), indicating the opposite frequency dependence of $\Gamma$ : the higherfrequency radiation is amplified more strongly. (Note that despite the absence of the standard giant pulses, it is still possible to notice the considerable intensity amplification in this pulsar). On the whole, for any pulsar the frequency behaviour of the scattering efficiency over a broad frequency range can hardly be presented as a single power law and therefore various pairs of frequencies can yield different $l$.

\section{Conclusions}

We have considered the induced Compton scattering of radio emission off the particles of ultrarelativistic highly magnetized electron-positron plasma in pulsars. It is shown that the scattering between two substantially different photon states results in photon transfer to the higher-frequency state. With the decreasing spectrum of pulsar radiation, this may imply a significant narrow-band amplification of the higher-frequency intensity, which is supposed to underlie the phenomenon of giant pulses.

As is known, only the photon states obeying the condition (2) can be involved in the induced scattering, so that for widely spaced frequencies the photon orientations should differ. In the pulsar case, different orientations of the rays of distinct frequencies can be attributed either to the radius-tofrequency mapping combined with the magnetosphere rotation or to the frequency-dependent refraction. Apparently, the difference in the orientations of the rays is the most pronounced if the higher-frequency rays are almost parallel to the local magnetic field. In the case of PSR B1937+21 this regime is found to be set up by the rotational effect and to take place in a certain region of the open field line tube; as a result, giant pulses should be confined to a narrow longitudinal interval determined roughly by the locus of the magnetic axis projection. Keeping in mind this conclusion, note the recently discovered coincidence of the giant pulse profiles with the peak of the X-ray profile (Cusumano et al. 2003) may give some insight into the nature of the high-energy emission mechanism in this pulsar. In the Crab pulsar, the regime of quasi-longitudinal propagation can be caused by refraction and happen at different locations in the tube, in which case giant pulses can be found throughout the pulse window. Note that among the recently discovered giant pulse sources, PSR B1821-24 and PSR B0540-69 are very similar to PSR B1937+21 and the Crab pulsar (e.g. Johnston $\&$ Romani 2003), and our consideration is applicable to these pulsars as well.

The efficiency of induced scattering depends on the (average) radio luminosity of a pulsar. Note that the two most prominent sources of giant pulses (the Crab pulsar and PSR B1937+21) have the largest known radio luminosities. Unfortunately, the optical depth to induced scattering appears to depend drastically on the location of the scattering region and the scattering geometry and therefore does not allow firm conclusions as to the possible candidates of the giant pulse sources. The possibility of giant pulses also depends on the intensity ratio of the beams participating in the beamto-beam scattering, which determines the maximum possible level of amplification of the higher-frequency intensity. A significant level of amplification is expected for the steep (average) spectrum of pulsar radiation. Note that the known sources of giant pulses have very steep radio spectra. On the whole, 
the physical parameters of giant pulse sources can scarcely be thought to differ qualitatively from those of other pulsars. They rather represent the combinations particularly favourable for strong beam-to-beam scattering. At the same time, the induced scattering is believed to be efficient in a number of pulsars, giving rise to various phenomena such as microstructure, giant micropulses, non-Gaussian tails of the distributions of pulses in intensity at certain pulse longitudes, etc.

The statistical behaviour of giant pulses can be explained within the framework of the simple model: the scattering efficiency varies because of pulse-to-pulse fluctuations of the particle number density and the incident intensity. Given that these quantities are considered as partially correlated Gaussian random quantities, the histogram of the intensities subject to substantial amplification $(\Gamma>1)$ has a power-law form, with the slope being determined by the characteristic optical depth and the parameters of the bivariate Gaussian distribution. Note that for the observed giant pulses the slope of the intensity distribution depends on the size of the sample studied and only at $N_{\text {total }} \rightarrow \infty$ approaches the theoretical value.

A non-linear intensity growth as a result of induced scattering implies that even huge amplification is caused by moderately large plasma densities and original intensities (within the order of magnitude relative to the average values). Therefore neither dramatic electrodynamic changes nor distinct emission mechanisms are required to account for giant pulses. Relative stability of the characteristics of the plasma flow is confirmed by the absence of essential variation in the highenergy emission concurrent with giant pulses (Lundgren et al. 1995; Shearer et al. 2003), whereas the lack of significant temporal delay of giant pulses of the Crab pulsar (Lundgren et al. 1995) testifies to the common emission mechanism for normal and giant pulses.

Because of a marked spatial dependence of the efficiency of induced scattering, giant pulses should be sufficiently narrow, $w_{\mathrm{GP}} \sim w / \Gamma$, especially the strongest ones, which correspond to large enough $\Gamma$. In addition, they may consist of separate components. Similarly to the microstructure in the normal pulsars, the substructure of the giant pulses can be attributed to the focusing effect as a result of induced scattering within a single narrow beam (for more details see Petrova 2004). Note that within the framework of this interpretation, the problem of exceedingly high brightness temperatures of the nanopulses (Hankins et al. 2003) is removed naturally. Besides that, based on the focusing effect one can explain the constancy of the energy contained in the brightest subpulses of various widths (Eilek et al. 2002) and anticipate the shorter timescales for the substructure of giant pulses in PSR B1937+21 (cf. Popov \& Stappers 2003).

Owing to the explicit frequency dependence of $\Gamma$, the bandwidth of the amplified radiation is sufficiently narrow, $\Delta v / v \sim$ $1 / \Gamma \gtrsim 0.1$, being compatible with the observed correlation bandwidth for the substructure in the Crab pulsar. At the same time, the conditions for efficient beam-to-beam scattering can be fulfilled at different locations in the magnetosphere and for different frequencies independently. This is believed to result in a complicated picture of giant pulse behaviour over a broad frequency range, which is yet to be studied. It should be noted that the histograms of the dual-frequency spectral indices of single pulses exhibit one-sided tails (Hankins 2000; Kramer et al. 2003), which can indeed be interpreted as a signature of frequency-dependent amplification.

On the whole, the main features of the observed giant pulse phenomenon can be explained in terms of induced Compton scattering of pulsar radio emission off the plasma particles.

Acknowledgements. This research is in part supported by INTAS Grant (Call 2003).

\section{References}

Asseo, E., Pelletier, G., \& Sol, H. 1990, MNRAS, 247, 529

Blandford, R. D., \& Scharlemann, E. T. 1976, MNRAS, 174, 59

Cognard, I., Shrauner, J. A., Taylor, J. H., \& Thorsett, S. E. 1996, ApJ, 457, L81

Cordes, J. M., Bhat, N. D. R., Hankins, T. H., McLaughlin, M. A., \& Kern, J. 2003 [arXiv: astro-ph/0304495]

Cusumano, G., Hermsen, W., Kramer, M., et al. 2003, A\&A, 410, L9

Eilek, J. A., Arendt, P. N., Hankins, T. H., \& Weatherall, J. C. 2002, in Neutron Stars, Pulsars and SNRs, ed. W. Backer, H. Lesch, \& J. Trumper, MPE Rep., N278, 249

Ershov, A. A., \& Kuzmin, A. D. 2003, Astron. Lett., 29, 91

Gradshteyn, I. S., \& Ryzhik, I. M. 1980, Tables of Integrals, Series and Products (New York: Academic Press)

Gupta, Y., Bhat, N. D. R., \& Rao, A. P. 1999, ApJ, 520, 173

Hankins, T. H. 1996, in Pulsars: Problems \& Progress, ed. S. Johnston, M. A. Walker, \& M. Bailes, ASP Conf. Ser., 105, 197

Hankins, T. H. 2000, in Proc. IAU Coll., 177, Pulsar Astronomy 2000 and Beyond, ed. M. Kramer, N. Wex, \& R. Wielebinsky (San Francisco: ASP), ASP Conf. Ser., 202, 165

Hankins, T. H., Kern, J. S., Weatherall, J. C., \& Eilek, J. A. 2003, Nature, 422, 141

Hibschman, J. A., \& Arons, J. 2001a, ApJ, 554, 624

Hibschman, J. A., \& Arons, J. 2001b, ApJ, 560, 871

Johnston, S., \& Romani, R. 2003, ApJ, 590, 95

Kinkhabwala, A., \& Thorsett, S. E. 2000, ApJ, 535, 365

Kramer, M., Karastergiou, A., Gupta, Y., et al. 2003, A\&A, 407, 655

Kuzmin, A. D., Ershov, A. A., \& Losovsky, B. Ja. 2004, Astron. Lett., 30, 247

Lundgren, S. C., Cordes, J. M., Ulmer, M., et al. 1995, ApJ, 453, 433

Lyubarskii, Yu. E., \& Petrova, S. A. 1996, Astron. Lett., 22, 399

Malov, I. F., \& Malov, O. I. 1997, Astron. Rep., 41, 56

Petrova, S. A. 2000, A\&A, 360, 592

Petrova, S. A. 2001a, MNRAS, 324, 931

Petrova, S. A. 2001b, A\&A, 378, 883

Petrova, S. A. 2004, A\&A, 417, L29

Popov, M. V., Bartel, N., Cannon, W. H., et al. 2002, A\&A, 396, 171

Popov, M. V., \& Stappers, B. 2003, Astron. Rep., 47, 660

Rankin, J. M. 1983, ApJ, 274, 333

Romani, R., \& Johnston, S. 2001, ApJ, 557, 93

Sallmen, S., Backer, D. C., Hankins, T. H., Moffet, D., \& Lundgren, S. 1999, ApJ, 517, 460

Shearer, A., Stappers, B., \& Connor, P. O. 2003, Science, 301, 493

Taylor, J. H., Manchester, R. N., \& Lyne, A. G. 1993, ApJS, 88, 529

Weatherall, J. C. 1998, ApJ, 506, 341

Wolszczan, A., Bartlett, J. E., \& Cordes, J. M. 1988, in Radiowave scattering in the interstellar medium, ed. J. M. Cordes, B. J. Rickett, \& D. C. Backer (New York: AIP), AIP Conf. Proc., 174, 145 